\title{
Theory of pairing in the Cu-O Plane: Three-Band Hubbard Model and Beyond
}

\author{
Michele Cini, Gianluca Stefanucci and Adalberto Balzarotti \\ INFM, Dipartimento di Fisica, Università di Roma Tor Vergata, Via \\ della Ricerca Scientifica 1- 00133 Roma, Italy
}

(October 31, 2018)

\begin{abstract}
We calculate the effective interaction $W_{\text {eff }}$ between two holes added to the ground state of the repulsive three-band Hubbard model. To make contact with Cooper theory and with earlier Hubbard model cluster studies, we first use a perturbative canonical transformation, to generate a two-body Hamiltonian. Then, we extend the results to all orders. The approach is exact in principle, and we obtain a close analytic expression including explicitly the effects of all virtual transitions to 4-body intermediate states. Our scheme naturally lends itself to embody off-site, inter-planar, phonon-mediated and other interactions which are not considered in the Hubbard model but may well be important. The result depends qualitatively on the symmetry of the two-hole state: ${ }^{1} B_{2}$ and ${ }^{1} A_{2}$ pairs are special, because the bare holes do not interact by the on-site repulsion ( $\mathrm{W}=0$ pairs). The effective interaction in these channels is attractive and leads to a Cooper-like instability of the Fermi liquid; however $W_{\text {eff }}$ is repulsive for Triplet pairs. Bound two-hole states of the same nature were reported earlier in small cluster calculations by exact diagonalisation methods; only symmetric clusters are good models of the plane. Once $W_{e f f}$ is known, the pair eigenfunction is determined by an integral equation. We present numerical estimates of the binding energy $|\Delta|$ of the pairs, which is in the physically interesting range of tens of $\mathrm{meV}$ if unscreened on-site repulsion parameters are used.
\end{abstract}




\section{INTRODUCTION}

Cooper pairs in high- $T_{C}$ superconductors [1] are more tighly bound and spatially localized than in ordinary BCS ones, and this happens in the presence of strong correlations. If phonons are mainly responsible for the mechanism, an enhanced electron-phonon vertex must be involved [2] and a polaron or bipolaron behavior [3] is also a serious possibility. However the wave function of the hole system must be such that the repulsion barrier is minimized and can be overcome by phonon exchange. Several Authors have pushed the argument further, proposing that correlation effects alone could be so strong to turn the repulsion into attraction by some sort of overscreening and lead to pairing. The proposed electronic mechanisms have often been based on Hubbard [4,5] or t-J models [6], just to cite a few of the less exotic proposals. The phonon-based and electronic mechanisms have usually been regarded as alternative, but there is a clear need for a theoretical framework where they can coexist and can be compared on equal footing; in our opinion, all the serious mechanisms must convey part of the physics.

Our first aim here is to propose such a scheme, starting with the three-band Hubbard model. The Hamiltonian is

$$
H=H_{0}+W
$$

and the independent hole term reads, in the site representation

$$
H_{0}=\sum_{C u} \varepsilon_{d} n_{d}+\sum_{O} \varepsilon_{p} n_{p}+t \sum_{n . n .}\left[c_{p}^{\dagger} c_{d}+h . c .\right]
$$

where n.n. stands for nearest neighbors. The on-site repulsion term will be denoted by

$$
W=\sum_{i} U_{i} n_{i+} n_{i-}
$$

where $U_{i}=U_{d}$ for a $\mathrm{Cu}$ site, $U_{i}=U_{p}$ for an $\mathrm{O}$.

Several years ago, pioneers in cluster calculations 79 explored the possibility that pairing could result from repulsion. They proposed the following definition [7] of the energy of the pair:

$$
\Delta=E(N+2)+E(N)-2 E(N+1),
$$

where $E(N)$ is the ground state energy of the cluster with $N$ holes, as obtained by exact diagonalisation. A negative $\Delta$ means that the ionisation potential decreases with increasing the number of holes, and this may be taken as an indication of pairing. Several clusters were studied for $N=2$. It turned out that $\Delta<0$ is possible only if the off-site $U$ between $\mathrm{Cu}$ and $\mathrm{O}$ is taken to be unphysically large (about $5 \mathrm{eV}$ ). Moreover, the physical interpretation was not obviuos, and there was no clearcut reason for excluding hole bags. Also, interestingly,
S. Mazumdar et al. 10] argued that $\Delta<0$ could be an artifact due to the neglect of the degrees of freedom of the nuclei: when they are left free to move, the state with $N+1$ holes could gain enough energy from Jahn-Teller distortion that $\Delta$ could turn out to be positive, after all. Later, we pointed out [1] that a couple of key ingredients were missing, namely, symmetry and $\mathrm{W}=0$ pairs. $\mathrm{W}=0$ pairs are two-hole eigenstates of the kinetic energy $H_{0}$ that are also eigenstates of the on-site repulsion term $W$ with eigenvalue 0 . There is no on-site repulsion barrier to overcome in $\mathrm{W}=0$ pairs.

The symmetry of the cluster is essential, because only clusters with the same point symmetry group $C_{4 v}$ as the plane and centered on a $\mathrm{Cu}$ ion (fully symmetric clusters) allow such solutions. Still, also the planar lattice structure is essential, because no $\mathrm{W}=0$ pairs occur in $3 \mathrm{D}$ or in a continuous model, where the interaction term (3) becomes a contact term. We started [11] by the above definition of $\Delta$, and studied the fully symmetric clusters with up to 21 atoms by exact diagonalisation; $\Delta$ was found to be negative when (and only when) the least bound holes formed a $\mathrm{W}=0$ pair. The Hamiltonian had been parametrised by electron spectroscopy studies 12] and ab-initio calculations [13], and we used literature values. We also considered first-neighbor off-site interactions 11]. The O-O hopping can change the order of single-hole levels, thereby changing the occupation number which is necessary to get a partially occupied degenerate state; however, when the conditions are satisfied, the $\Delta<0$ behavior results without important modifications. The first neighbor off-site $\mathrm{Cu}-\mathrm{O}$ interactions were found to enhance the effect; the first neighbor off-site $\mathrm{O}$ $\mathrm{O}$ interactions unpair it, but their effect is small if the recommended values of the parameters are used. In the present paper, such terms are dropped for the sake of simplicity, since they are not essential for our present scope. Recent calculations by Schüttler et al [14], based on a combination of diagrammatic and Quantum Monte Carlo methods on an Extended Hubbard Model, support the present view that the screened interaction is attractive and the attraction is robust against the long-range part of the Coulomb repulsion.

In the fully symmetric $\mathrm{Cu}-\mathrm{O}$ clusters a genuine pairing takes place, due to an effective interaction which is attractive for singlets and repulsive for triplets. Let us summarize why this conclusion is free from the ambiguity pointed out in Ref. [10].

First, we computed $\Delta$ for our clusters by second-order ground state energy diagrams (modified for degenerate ground states, when appropriate) [15]. It is convenient to denote the hole orbitals by their symmetry labels, with $b$ for $b_{1}$ and $a,\left(a^{\prime}\right)$ for the occupied (empty) orbitals of $a_{1}$ symmetry: we obtained

$$
\Delta^{(2)}=-2\left[\sum_{b} \frac{W(a, b, x, x)^{2}}{\left(\varepsilon_{b}-\varepsilon_{a}\right)}-\sum_{a^{\prime}} \frac{W\left(a, a^{\prime}, x, x\right)^{2}}{\left(\varepsilon_{a^{\prime}}-\varepsilon_{a}\right)}\right],
$$


where $\varepsilon_{a}$ is the one-hole energy of the $a$ orbital and so on; the sums run over all empty states of the appropriate symmetries and involve the matrix elements of the onsite interaction $W$. No contributions arise to second order from the empty states of $e$ symmetry since the relevant matrix elements vanish.

Second, we computed to second-order the two-hole amplitude for holes of opposite spins in the degenerate $(x, y)$ orbitals. The only contributions are the second-order diagrams of Figure 1.

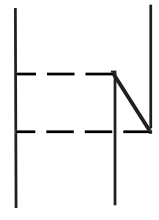

a)

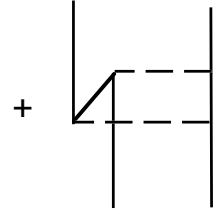

b)
FIG. 1. The second order diagrams for the Two-Hole Amplitude.

The system makes virtual transitions to 4-body (3 holes and 1 electron) states. We demonstrated that this produced an effective interaction, which pushes down the singlet and up the triplet by $\left|\Delta^{(2)}\right|$. In this way, $\Delta$ can be redefined without any reference to the ground state of clusters with a different number of holes, and we are free from the objections based on the Jahn-Teller distortion of odd- $N$ clusters. Good agreement between the second-order calculation and the numerical exact diagonalisation results supported the interpretation. Thus, the cluster calculations 16 18 showed that $\mathrm{W}=0$ pairs are the "bare" quasiparticles that, when "dressed", become a bound state. That approach is inherently limited by the small size of solvable clusters, but allows a very explicit display of paired hole properties, that even show superconducting flux-quantization [15,19]. Our second task here is to extend the theory to the full plane, following the hint provided by the diagrams. In Sect.II we review the one-body properties of the model, in order to estabilish some notation and several useful formulas. In Sect.III we show how $\mathrm{W}=0$ pairs in the full plane can be obtained for any filling. The effective interaction between holes is calculated by second-order perturbation theory in Sect. IV, with the aim of showing the relation of the present formalism to Cooper theory from one side and to the cluster results from the other. With this preparation, we shall be ready to present in Sect. $V$ the general canonical transformation leading to the effective Hamiltonian for pairs [20]. This is free from the limitations of perturbation theory and differs from the results of Sect. IV in an important way, although it is seen to reduce to them in the appropriate limit. It will turn out that the effective interactions are additive, and the theory is able to accommodate the effects of phonons, off-site interactions [11], interplanar coupling 21 and whatever physics one wants to include. The final result is an integral equation for pairs in the full plane, which is suitable for a numerical study. Sect. VI is devoted to the method we used to solve the integral equation in finite supercells and than taking the asymptotic limit. Two kinds of bound states of different symmetries result, and Sect. VII presents the dependence of $\Delta$ on the filling and other parameters. Finally, Sect. VIII summarizes our conclusions.

\section{BAND STRUCTURE}

The eigenstates of

$$
H_{0} \psi^{(\nu)}(k, r)=\varepsilon^{(\nu)}(k) \psi^{(\nu)}(k, r)
$$

where $\nu$ is a band index, are written according to Bloch's theorem

$$
\psi^{(\nu)}(k, r)=e^{i k r} \phi^{(\nu)}(k, r),
$$

where $\phi$ is periodic. If $r=0, r=a$ and $r=b$ are the positions of $\mathrm{Cu}$ and of the two Oxygens, respectively, the non-bonding band is characterized by $\phi^{(n b)}(k, 0)=0$; one then finds

$$
\begin{aligned}
\phi^{(n b)}(k, a) & =\frac{\cos \left[\frac{k_{x} d}{2}\right]}{\sqrt{N_{C}\left(\cos ^{2}\left[\frac{k_{x} d}{2}\right]+\cos ^{2}\left[\frac{k_{y} d}{2}\right]\right)}}, \\
\phi^{(n b)}(k, b) & =\frac{\cos \left[\frac{k_{y} d}{2}\right]}{\sqrt{N_{C}\left(\cos ^{2}\left[\frac{k_{x} d}{2}\right]+\cos ^{2}\left[\frac{k_{y} d}{2}\right]\right)}},
\end{aligned}
$$

where $N_{C}$ is the number of cells in the crystal and $d$ the lattice parameter. For the other bands, one obtains the eigenvalue equation

$$
\varepsilon^{( \pm)}(k)=\frac{\varepsilon_{p} \pm r}{2}
$$

( + for the antibonding and - for the bonding band), with

$$
r=\sqrt{\varepsilon_{p}^{2}+16 t^{2}\left[\cos ^{2}\left[\frac{k_{x} d}{2}\right]+\cos ^{2}\left[\frac{k_{y} d}{2}\right]\right]}
$$

and the $\mathrm{Cu}$ amplitudes 


$$
\phi^{( \pm)}(k, 0)=\sqrt{\frac{\varepsilon^{( \pm)}-\varepsilon_{p}}{N_{C}\left(2 \varepsilon^{( \pm)}-\varepsilon_{p}\right)}},
$$

while the $\mathrm{O}$ amplitudes are obtained from

$$
\begin{array}{r}
\left(\varepsilon_{p}-\varepsilon^{(\nu)}(k)\right) \phi^{(\nu)}(k, a) \\
+2 t \cos \left[\frac{k_{y} d}{2}\right] \phi^{(\nu)}(k, 0)=0,
\end{array}
$$

and

$$
\begin{array}{r}
\left(\varepsilon_{p}-\varepsilon^{(\nu)}(k)\right) \phi^{(\nu)}(k, b) \\
+2 t \cos \left[\frac{k_{x} d}{2}\right] \phi^{(\nu)}(k, 0)=0 .
\end{array}
$$

Setting $x=k_{x} d, y=k_{y} d$, we get the contours of constant energy $\varepsilon$ in the Brillouin Zone (BZ):

$$
\begin{gathered}
\cos (x)+\cos (y)=q(\varepsilon), \\
q(\varepsilon)=\frac{\varepsilon-\varepsilon_{p}}{2 t^{2}}-2 .
\end{gathered}
$$

In the bonding band, $q=2$ at the bottom and $q=-2$ at the top; at $q=0$ the contour is a square with vertices at $\frac{2 \pi}{d}( \pm 1,0)$ and $\frac{2 \pi}{d}(0, \pm 1)$, where the Fermi velocity vanishes. Let $D$ be the domain defined by $\varepsilon<E_{F}$ in the BZ.

Below half filling,

$$
\iint_{D} d x d y=\int_{a(0)}^{-a(0)} d x \int_{a(x)}^{-a(x)} d y
$$

where $a(x)=\arccos \left(q\left(E_{F}\right)-\cos (x)\right)$; at half filling

$$
\iint_{D} d x d y=\int_{-\pi}^{\pi} d x \int_{-\pi+|x|}^{\pi-|x|} d y
$$

and above half filling, putting $b(x)=\arccos \left(q\left(E_{F}\right)+\right.$ $\cos (x))$, we find

$$
\iint_{D} d x d y=I_{1}+I_{2}+I_{3}
$$

where

$$
\begin{gathered}
I_{1}=\int_{-b(0)}^{b(0)} d x \int_{-\pi}^{\pi} d y \\
I_{2}=\int_{-\pi}^{-b(0)} d x \int_{-a(x)}^{a(x)} d y,
\end{gathered}
$$

and

$$
I_{3}=\int_{b(0)}^{\pi} d x \int_{-a(x)}^{a(x)} d y
$$

The number of holes per spin per unit cell below $E_{F}$ in the bonding band above half filling is

$$
N\left(E_{F}\right)=\left(\frac{d}{2 \pi}\right)^{2} \iint d^{2} k \theta\left(E_{F}-\varepsilon(k)\right)
$$

differentiating, one finds the density of states

$$
\rho(\varepsilon)=\frac{\varepsilon_{p}-2 \varepsilon}{2 \pi^{2} t^{2}} \int_{\arccos [q+1]}^{\pi} \frac{d x}{\sqrt{1-(q-\cos (x))^{2}}},
$$

where $q=q(\varepsilon)$. Taking care of the singularity of the integrand at the lower limit, this expression is convenient for the numerical evaluation, and shows a logarithmic singularity at half filling (Van Hove singularity).

\section{III. $\mathrm{W}=0$ PAIRS}

We change representation with

$$
c_{\sigma}^{\dagger}\left(r_{i}\right)=\sum_{k, \nu} c_{k, \nu, \sigma}^{\dagger} \psi_{\sigma}^{(\nu) *}\left(k, r_{i}\right)
$$

where $k$ runs over the $\mathrm{BZ}$ and $\nu$ over bands. Thus,

$$
\begin{array}{r}
W=\sum_{k_{1} . . k_{4}} \sum_{\nu_{1} . . \nu_{4}} c_{k_{1}, \nu_{1},+}^{\dagger} c_{k_{2}, \nu_{2},+} c_{k_{3}, \nu_{3},-}^{\dagger} c_{k_{4}, \nu_{4},-} \\
\times U^{\left(\nu_{1} . . \nu_{4}\right)}\left(k_{1}, k_{3}, k_{2}, k_{4}\right),
\end{array}
$$

Using a shorthand notation, where the band indices are understood,

$$
\begin{aligned}
& U(m, n, p, q)=\sum_{i} e^{-i(m+n-p-q) r_{i}} \\
& \times \phi^{*}(m, i) \phi^{*}(n, i) \phi(p, i) \phi(q, i),
\end{aligned}
$$

is the lattice Fourier transform of a periodic function; the momenta are limited to the BZ, so, introducing reciprocal lattice vectors $G$ we may write

$$
\begin{array}{r}
U(m, n, p, q)=N_{C} \sum_{G} \delta( \\
\quad m+n-p-q-G) \\
\times U^{C}(m, n, p, q ; G),
\end{array}
$$

where $U^{C}$ involves a sum over a single cell:

$$
\begin{aligned}
& U^{C}(m, n, p, q ; G)=\sum_{i}^{\text {Cell }} U_{i} e^{-i G r_{i}} \\
& \times \phi(m, i) \phi(n, i) \phi(p, i) \phi(q, i),
\end{aligned}
$$

where stars have been dropped since $\phi^{\prime} s$ are real.The umklapp processes involving the basis vectors of the reciprocal lattice produce a - sign on one of the Oxygens. The matrix elements (28) are proportional to $N_{C}^{-1}$, like any bona fide two-body interaction.

Still omitting the band indices, we shall mean 


$$
d[k]=\left\|k_{+},-k_{-}\right\|=c_{k,+}^{\dagger} c_{-k,-}^{\dagger} \mid v a c>
$$

to be a two-hole determinantal state derived from the $k$ eigenfunctions. Since $\phi(-k, r)=\phi(k, r)$, which is required by time-reversal symmetry, the combination $d[k]+d[-k]$ is singlet and $d[k]-d[-k]$ is triplet.

The point symmetry Group of the $\mathrm{Cu}-\mathrm{O}$ plane is $C_{4 v}$, and its character Table is shown in Table I.

We introduce the determinants $R d[k]=d[R k]=$ $d\left[k_{R}\right], R \in C_{4 v}$, and the projected states

$$
\Phi_{\eta}[k]=\frac{1}{\sqrt{8}} \sum_{R \in C_{4 v}} \chi^{(\eta)}(R) R d[k]
$$

where $\chi^{(\eta)}(R)$ is the character of the operation $R$ in the Irreducible Representation (Irrep) $\eta$. In the nondegenerate Irreps, the operations that produce opposite $k_{R}$ have the same character, and the corresponding projections lead to singlets. Let $R_{i}, i=1, . .8$ denote the operations of $C_{4 v}$ and $k, k^{\prime}$ any two points in the BZ. Consider any two-body operator $\hat{O}$, which is symmetric $\left(R_{i}^{\dagger} \hat{O} R_{i}=\hat{O}\right)$, and the matrix with elements $O_{i, j}=<d[k]\left|R_{i}^{\dagger} \hat{O} R_{j}\right| d\left[k^{\prime}\right]>$, where $k$ and $k^{\prime}$ may be taken to be in the same or in different bands. This matrix can be diagonalized by Group Theory. Indeed, for each Irrep $\eta$, consider the normalized vector with components $\frac{1}{\sqrt{8}} \chi^{(\eta)}\left(R_{i}\right)$; this is an eigenvector of the operator matrix $R_{i}^{\dagger} \hat{O} R_{j}$, as we can check by noting that $\frac{1}{8} \sum_{i, j} \chi^{(\alpha)}\left(R_{i}\right) R_{i}^{-1} \hat{O} R_{j} \chi^{(\beta)}\left(R_{j}\right)=\hat{O} P^{2}(\alpha) \delta(\alpha, \beta) ;$ hence, $R_{i}^{\dagger} \hat{O} R_{j}$ is diagonal on the basis of the symmetry projected states. The square of the projection operator is $P^{2}(\alpha)=\sqrt{8} P(\alpha)$. Now, taking the matrix element between the $k$ and $k^{\prime}$ determinants, we get the eigenvalues in terms of determinantal matrix elements:

$$
O\left(\eta, k, k^{\prime}\right)=\sum_{R} \chi^{(\eta)}(R) O_{R}\left(k, k^{\prime}\right)
$$

where

$$
O_{R}\left(k, k^{\prime}\right)=\left\langle d[k]|\hat{O}| R d\left[k^{\prime}\right]\right\rangle .
$$

Thus, omitting the $k, k^{\prime}$ arguments, we get

$$
\begin{array}{r}
O\left({ }^{1} A_{1}\right)= \\
+O_{E}+O_{C_{2}}+O_{C_{4}}+O_{C_{4}^{3}} \\
+O_{\sigma_{x}}+O_{\sigma_{y}}+O_{\sigma_{1}^{\prime}}+O_{\sigma_{2}^{\prime}} \\
\begin{aligned}
O\left({ }^{1} A_{2}\right)= & O_{E}+O_{C_{2}}+O_{C_{4}}+O_{C_{4}^{3}} \\
& -O_{\sigma_{x}}-O_{\sigma_{y}}-O_{\sigma_{1}^{\prime}}-O_{\sigma_{2}^{\prime}} \\
O\left({ }^{1} B_{2}\right)= & O_{E}+O_{C_{2}}-O_{C_{4}}-O_{C_{4}^{3}} \\
& -O_{\sigma_{x}}-O_{\sigma_{y}}+O_{\sigma_{1}^{\prime}}+O_{\sigma_{2}^{\prime}}
\end{aligned}
\end{array}
$$

and for the $x$ component of the degenerate triplet

$$
O\left({ }^{3} E_{x}\right)=O_{E}-O_{C_{2}}+O_{\sigma_{x}}-O_{\sigma_{y}} .
$$

Triplets of the other Irreps are obtained from determinants of the form $\left\|k_{1}, k_{2}\right\|$, but they vanish when $k_{2}=-k_{1}$. If $\hat{O}$ is identified with $W$, the determinantal matrix element does not depend on the sign of the components of $k^{\prime}$, because the $\phi^{\prime} s$ depend on $k$ only through a cosine. The only thing that matters is that $C_{4}, C_{4}^{3}, \sigma_{1}^{\prime}$ and $\sigma_{2}^{\prime}$ exchange $k_{x}^{\prime}$ and $k_{y}^{\prime}$, while the other operations leave them in place. Consequently, $W_{E}=W_{C_{2}}=W_{\sigma_{x}}=W_{\sigma_{y}}$ and $W_{C_{4}}=W_{C_{4}^{3}}=W_{\sigma_{1}^{\prime}}=W_{\sigma_{2}^{\prime}}$, and

$$
W\left({ }^{1} A_{2}\right)=W\left({ }^{1} B_{2}\right)=0 .
$$

These are $\mathrm{W}=0$ pairs, like those studied previously [15], but with an important change, since in the cluster calculations the symmetries of $\mathrm{W}=0$ pairs were found [19] to be ${ }^{1} B_{2}$ and ${ }^{1} A_{1}$. The reason for this change is a twofold size effect. On one hand, ${ }^{1} A_{1}$ pairs have the $\mathrm{W}=0$ property only in the small clusters, having the topology of a cross, and belonging to the $S_{4}$ Group, but do not generalize as such to the full plane, when the symmetry is lowered to $C_{4 v}$; on the other hand, the small clusters admit no solutions of ${ }^{1} A_{2}$ symmetry at all.

One necessary condition for pairing in clusters is that the least bound holes form such a pair, and this dictates conditions on the occupation number. In the full plane, however, $\mathrm{W}=0$ pairs exist at the Fermi level for any filling. The explicit $\mathrm{W}=0$ singlet pair states are:

$$
\begin{array}{r}
\Phi_{1_{B_{2}}}\left[k, r_{1}, r_{2}\right]= \\
-\frac{\chi_{0}}{\sqrt{2}}\left\{\cos \left[k\left(r_{1}-r_{2}\right)\right] \phi\left(k, r_{1}\right) \phi\left(k, r_{2}\right)\right. \\
-\cos \left[k_{C_{4}}\left(r_{1}-r_{2}\right)\right] \phi\left(k_{C_{4}}, r_{1}\right) \phi\left(k_{C_{4}}, r_{2}\right) \\
-\cos \left[k_{\sigma}\left(r_{1}-r_{2}\right)\right] \phi\left(k_{\sigma}, r_{1}\right) \phi\left(k_{\sigma}, r_{2}\right) \\
\left.+\cos \left[k_{\sigma^{\prime}}\left(r_{1}-r_{2}\right)\right] \phi\left(k_{\sigma^{\prime}}, r_{1}\right) \phi\left(k_{\sigma^{\prime}}, r_{2}\right)\right\}
\end{array}
$$

and

$$
\begin{array}{r}
\Phi_{{ }^{A_{2}}}\left[k, r_{1}, r_{2}\right]= \\
+\operatorname{\chi _{0}} \frac{}{\sqrt{2}}\left\{\cos \left[k\left(r_{1}-r_{2}\right)\right] \phi\left(k, r_{1}\right) \phi\left(k, r_{2}\right)\right. \\
+\cos \left[k_{C_{4}}\left(r_{1}-r_{2}\right)\right] \phi\left(k_{C_{4}}, r_{1}\right) \phi\left(k_{C_{4}}, r_{2}\right) \\
-\cos \left[k_{\sigma}\left(r_{1}-r_{2}\right)\right] \phi\left(k_{\sigma}, r_{1}\right) \phi\left(k_{\sigma}, r_{2}\right) \\
\left.-\cos \left[k_{\sigma^{\prime}}\left(r_{1}-r_{2}\right)\right] \phi\left(k_{\sigma^{\prime}}, r_{1}\right) \phi\left(k_{\sigma^{\prime}}, r_{2}\right)\right\}
\end{array}
$$

where $\chi_{0}$ is a singlet spin function. Using the above explicit Bloch states we can verify that both vanish for $r_{1}=r_{2}$. This is another way to see that they have the $\mathrm{W}=0$ property. All the distinct $\mathrm{W}=0$ pairs of each symmetry may be labeled from the $k$ points in $1 / 8$ of the empty part of the BZ: for instance, those with $k_{x}>k_{y}>0$. We shall denote such a set by $e / 8$.

\section{CANONICAL TRANSFORMATION-SECOND ORDER}

In this Section, we shall parallel as closely as possible the Cooper theory 22 where an effective interaction involving phonons is introduced via an approximate canonical transformation. The treatment we give here 
is based on second-order perturbation theory and is not yet adequate for the present purposes, but is useful as a prelude to the more complete theory given in the next Section. We wish to stress the formal analogy with the BCS theory and make contact with the $\Delta$ expression derived from cluster studies. Suppose we add two holes to a background Fermi sphere. We use Roman indices for the zeroth-order pair states, which satisfy

$$
H_{0}|m\rangle=E_{m}|m\rangle
$$

with $E_{m}=2 \varepsilon(m)$. The basic idea here is that of obtaining an effective interaction for two holes using the process of the diagrams in Fig.1. Accordingly, the pair states are coupled to the set of 3 hole- 1 electron intermediate states, which satisfies

$$
H_{0}|\alpha\rangle=E_{\alpha}|\alpha\rangle \text {. }
$$

Thus, the effect of the perturbation is of the form

$$
W|m\rangle=\sum_{m^{\prime}}\left|m^{\prime}\right\rangle W_{m^{\prime}, m}+\sum_{\alpha}|\alpha\rangle W_{\alpha, m}
$$

where $W_{m^{\prime}, m}$ stands for the interaction between pairs, while $\alpha$ is the set of 3 hole- 1 electron intermediate states which are coupled to the pairs by the diagram;

$$
W|\alpha\rangle=\sum_{m}|m\rangle W_{m, \alpha},
$$

The off-diagonal $W_{m^{\prime}, m}$ elements vanish for $\mathrm{W}=0$ pairs; on the other hand, in the many-body problem, the diagonal elements $W_{m, m}$ do not vanish, because of the tadpole-diagram contributions to the self-energy of the holes. However, the diagonal terms do not contribute to the effective interaction, but simply renormalize the $\varepsilon$ parameters in $H_{0}$. We had already met these tadpole diagrams in the calculation of $\Delta^{(2)}$ for clusters [15] where they were seen to cancel in the calculation of (4). Anyway, we keep $W_{m^{\prime}, m}$ in (43) which allows to introduce the effects of other interactions which are not included in the Hubbard model. In the present Section we shall consider $W$ as a small operator and look for an approximate canonical transformation such that the new Hamiltonian $\tilde{H}$ decouples the $\alpha$ states to first order; then, $\tilde{H}$ operates on the space of pairs. In other terms, we seek a first-order anti-Hermitean operator $\Lambda$ such that

$$
\tilde{H}=e^{-\Lambda} H e^{\Lambda}
$$

has no linear term connecting the $|\alpha\rangle$ states with the $|m\rangle$ states. Since

$$
\tilde{H}=H+[H, \Lambda]+\frac{1}{2}[[H, \Lambda], \Lambda]+\varnothing\left(\Lambda^{3}\right)
$$

expands to read

$$
\tilde{H}=H+\left[H_{0}, \Lambda\right]+[W, \Lambda]+\frac{1}{2}\left[\left[H_{0}, \Lambda\right], \Lambda\right]+\ldots
$$

this is accomplished if

$$
W_{\alpha, m}+\left(E_{\alpha}-E_{m}\right) \Lambda_{\alpha, m}=0
$$

with all others $\Lambda$-matrix elements equal to zero.

Assuming that the denominators do not vanish (more about that later) we obtain

$$
\langle\alpha|\Lambda| m\rangle=\frac{\langle\alpha|W| m\rangle}{E_{m}-E_{\alpha}}
$$

In $\tilde{H}$, the holes interact through the effective vertex of Figure 2 .

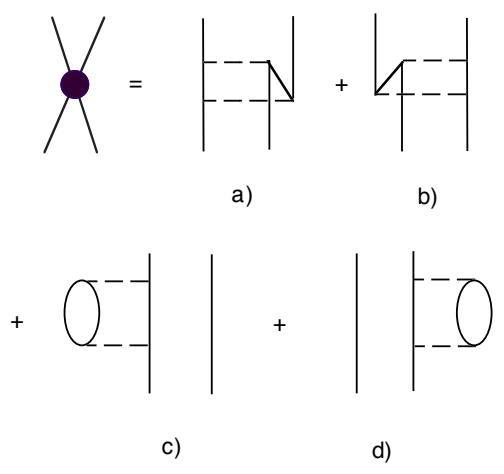

FIG. 2. The effective hole-hole vertex (blob) defined by the second-oder $\tilde{H}$. The dashed lines represent $W$ interactions. Linked diagrams belong to $\tilde{W}_{\text {eff }}$, and unlinked ones to $F$.

The interaction lines in the right hand side represent $W$; while a) and its specular counterpart b) correspond to the effective interaction diagrams of Figure 1, c) and d) are unlinked and represent self-energy processes of each hole. The same situation occurs in Cooper theory, where the self-energy processes involve the emission and absorption of a phonon by the same electron.

We may write

$$
\tilde{H}=H_{0}+F+\tilde{W}_{e f f}
$$

where $F$ is diagonal in the pair space, like $H_{0}$, and corresponds to the unlinked self-energy diagrams, while the effective interaction operator is $\tilde{W}_{\text {eff }}$. Like in Cooper theory, $F$ will be dropped. Formally, we write

$$
F+\tilde{W}_{e f f}=\frac{1}{2}[W, \Lambda]
$$

and

$$
\begin{array}{r}
2\left\langle m\left|F+\tilde{W}_{e f f}\right| m^{\prime}\right\rangle \\
\sum_{\alpha} W_{m, \alpha} W_{\alpha, m^{\prime}}\left[\frac{1}{E_{m}-E_{\alpha}}+\frac{1}{E_{m^{\prime}}-E_{\alpha}}\right]
\end{array}
$$


The $\alpha$ states are 3 hole- 1 electron determinants which carry no quasi-momentum. We write

$$
|\alpha>=|\left\|\left(k^{\prime}+q+k_{2}\right)_{+}, \bar{k}_{2-},-q_{-},-k_{-}^{\prime}\right\|>
$$

where $\bar{k}_{2+}$ is the electron state and pedices refer to the spin direction; $\alpha$ states with opposite spin indices arise from the specular diagram, contribute similarly and yield a factor of 2 at the end. The unperturbed eigenvalues are:

$$
E_{\alpha}=\varepsilon\left(k^{\prime}+q+k_{2}\right)-\varepsilon\left(k_{2}\right)+\varepsilon(q)+\varepsilon\left(k^{\prime}\right) .
$$

Let us first do the calculation on a determinantal basis. We work out the interaction matrix element:

$$
\begin{array}{r}
<\left\|\left(k^{\prime}+q+k_{2}\right)_{+}, \bar{k}_{2-},-q_{-},-k_{-}^{\prime}\right\||W| d[s]>= \\
\delta(q-s) U\left(q+k^{\prime}+k_{2},-k^{\prime}, s, k_{2}\right) \\
-\delta\left(k^{\prime}-s\right) U\left(q+k^{\prime}+k_{2},-q, s, k_{2}\right)
\end{array}
$$

two of the 4 terms in the product in (52) are proportional to $\delta(p-s)$ and belong to $F$, while the cross terms yield identical contributions to the effective interaction. Symbolically, we may write

$$
\begin{aligned}
\left\langle m\left|\tilde{W}_{e f f}\right| m^{\prime}\right\rangle= & \frac{1}{2} \sum_{\alpha}\left[W_{m, \alpha} W_{\alpha, m^{\prime}}\right]_{\text {Linked }} \\
& {\left[\frac{1}{E_{m}-E_{\alpha}}+\frac{1}{E_{m^{\prime}}-E_{\alpha}}\right] }
\end{aligned}
$$

Including the specular diagram by a factor of 2 , we write the interaction between determinantal states (with $s$ and $p$ empty).

$$
\begin{array}{r}
\left\langle d[p]\left|\tilde{W}_{\text {eff }}\right| d[s]\right\rangle=2 \sum_{k}^{o c c} \theta\left(\varepsilon(s+p+k)-E_{F}\right) \\
\times U(s+p+k,-p, s, k) U(p, k, s+p+k,-s) \\
+\frac{1}{\varepsilon(s+p+k)-\varepsilon(k)-\varepsilon(s)+\varepsilon(p)} \\
\left.+\frac{1}{\varepsilon(s+p+k)-\varepsilon(k)-\varepsilon(p)+\varepsilon(s)}\right\} .
\end{array}
$$

The interaction between symmetry projected states is obtained using (32,33) and is given by

$$
\begin{array}{r}
\left\langle\Phi_{\alpha}[p]\left|\tilde{W}_{\text {eff }}\right| \Phi_{\alpha}[s]\right\rangle= \\
\sum_{R} \chi^{(\alpha)}(R)\left\langle d[p]\left|\tilde{W}_{\text {eff }}\right| R d[s]\right\rangle
\end{array}
$$

The above theory applies not only to the plane but also to clusters, provided that they are fully symmetric and allow $\mathrm{W}=0$ pairs. The natural basis set is provided by the orbitals, rather than the Bloch states; in the small clusters with open boundary conditions that we studied previously, pairing occurs when a degenerate state of the Irrep $e(x, y)$ is occupied by two holes. Then, in terms of orbitals, the only ${ }^{1} B_{2} \mathrm{~W}=0$ pair available for pairing is

$$
\Phi_{B_{2}}=\frac{\left\|x_{+} y_{-}\right\|+\left\|y_{+} x_{-}\right\|}{\sqrt{2}} .
$$

The diagonal element of $\tilde{W}_{\text {eff }}$ on this state gives the second-order energy shift of the pair; one calculates the $W$ matrix on the orbital basis and after some algebra one finds the above $\Delta^{(2)}$ expression (5). In the small clusters, the diagonal element provides the binding energy, while the localization is granted by the boundary conditions. In the full plane, there is a continuous infinity of $\mathrm{W}=0$ pairs for each of the allowed symmetries, and the off-diagonal elements are essential to form a localized wave packet. Therefore the localized state is not known a priori, and $\Delta$ cannot be obtained as an expectation value of $\tilde{W}_{\text {eff }}$, but must be found by solving a two-body problem with interaction $\tilde{W}_{\text {eff }}$ (See Sect.VI).

In the continuum limit, the integral in (58) must be understood as a principal part, because the denominators vanish in a set of vanishing measure within the integration domain. While in principle this is no trouble, the numerical evaluation is difficult. However, this computational difficulty is an artifact of the perturbation approach, as will become clear in the next Section.

Above all, the approximate canonical transformation is open to question because it assumes that $W$ be weak. In small clusters, it works remarkably well, even for at large couplings, but one could argue that the reason might be that $\Delta$ is still small compared to the average distance between the discrete levels. When the levels form a continuum, the applicability of perturbation theory is not obvious. Therefore, it is important to put the theory on a clearer and firmer basis, as we do in the next Section. A posteriori, it turns out that high order diagrams mererly renormalize some quantities without destroying the structure of the theory, because it is a property of $\mathrm{W}=0$ pairs that the interaction is dynamically small.

\section{CANONICAL TRANSFORMATION - ALL ORDERS}

Suppose the $\mathrm{Cu}-\mathrm{O}$ plane is in its ground state with Fermi energy $E_{F}$ and a couple of extra holes are added. We wish to show that by a canonical transformation [20] one obtains an effective Hamiltonian which describes the propagation of a pair of dressed holes, and includes all many-body effects.

The exact many-body ground state with two added holes may be expanded in terms of excitations over the vacuum (the non-interacting Fermi sphere) by a configuration interaction:

$$
\left|\Psi_{0}>=\sum_{m} a_{m}\right| m>+\sum_{\alpha} b_{\alpha}\left|\alpha>+\sum_{\beta} c_{\beta}\right| \beta>+\ldots .
$$

here $m$ runs over pair states, $\alpha$ over 4 -body states $(2$ holes and 1 e-h pair), $\beta$ over 6 -body ones ( 2 holes and 2 e-h pairs), and so on. To set up the Schrödinger equation, 
we consider the effects of the operators on the terms of $\mid \Psi_{0}>$. We write:

$$
H_{0}\left|m>=E_{m}\right| m>, H_{0}\left|\alpha>=E_{\alpha}\right| \alpha>, \ldots
$$

and since $W$ can create or destroy up to 2 e-h pairs,

$$
\begin{aligned}
W\left|m>=\sum_{m^{\prime}} W_{m^{\prime}, m}\right| m^{\prime}> & +\sum_{\alpha} \mid \alpha>W_{\alpha, m} \\
& +\sum_{\beta} \mid \beta>W_{\beta, m} .
\end{aligned}
$$

As explained in the previous Section, $W_{m^{\prime}, m}$ does not contribute to the effective interaction for $\mathrm{W}=0$ pairs in our model; however we keep it for generality, since it allows to introduce the effect of the exchange of phonons and other quasiparticles that we are not considering. For clarity let us first write the equations that include explicitly up to 6-body states; then we have

$$
\begin{aligned}
W\left|\alpha>=\sum_{m}\right| m>W_{m, \alpha} & +\sum_{\alpha^{\prime}} \mid \alpha^{\prime}>W_{\alpha^{\prime}, \alpha} \\
& +\sum_{\beta} \mid \beta>W_{\beta, \alpha}
\end{aligned}
$$

where scattering between 4-body states is allowed by the second term, and

$$
\begin{array}{r}
\left.W\left|\beta>=\sum_{m^{\prime}}\right| m^{\prime}\right\rangle W_{m^{\prime}, \beta}+\sum_{\alpha}|\alpha\rangle W_{\alpha, \beta} \\
+\sum_{\beta^{\prime}}\left|\beta^{\prime}\right\rangle W_{\beta^{\prime}, \beta}
\end{array}
$$

In principle, the $W_{\beta^{\prime}, \beta}$ term can be eliminated by taking linear combinations of the complete set of $\beta$ states: when this is done, we get a self-energy correction to $E_{\beta}$ and a mixing of the vertices, without altering the structure of the equations. The Schrödinger equation yields equations for the coefficients $a, b$ and $c$

$$
\begin{array}{r}
\left(E_{m}-E_{0}\right) a_{m} \\
+\sum_{m^{\prime}} a_{m^{\prime}} W_{m, m^{\prime}}+\sum_{\alpha} b_{\alpha} W_{m, \alpha}+\sum_{\beta} c_{\beta} W_{m, \beta}=0 \\
\left.+\sum_{m^{\prime}} a_{m^{\prime}} W_{\alpha, m^{\prime}}+\sum_{\alpha^{\prime}} b_{\alpha^{\prime}} W_{\alpha, \alpha^{\prime}}+\sum_{\beta} c_{\beta} W_{\alpha, \beta}\right) b_{\alpha}=0 \\
\left(E_{\beta}-E_{0}\right) c_{\beta}+\sum_{m^{\prime}} a_{m^{\prime}} W_{\beta, m^{\prime}}+\sum_{\alpha^{\prime}} b_{\alpha^{\prime}} W_{\beta, \alpha^{\prime}}=0
\end{array}
$$

where $E_{0}$ is the ground state energy. Then, we exactly decouple the 6 -body states by solving the equation for $c_{\beta}$ and substituting into (65, 66), getting:

$$
\begin{gathered}
\left(E_{m}-E_{0}\right) a_{m}+\sum_{m^{\prime}} a_{m^{\prime}}\left[W_{m, m^{\prime}}+\sum_{\beta} \frac{W_{m, \beta} W_{\beta, m^{\prime}}}{E_{0}-E_{\beta}}\right] \\
+\sum_{\alpha} b_{\alpha}\left[W_{m, \alpha}+\sum_{\beta} \frac{W_{m, \beta} W_{\beta, \alpha}}{E_{0}-E_{\beta}}\right]=0 \\
\left(E_{\alpha}-E_{0}\right) b_{\alpha}+\sum_{m^{\prime}} a_{m^{\prime}} W_{\alpha, m^{\prime}} \\
+\sum_{\alpha^{\prime}} b_{\alpha^{\prime}}\left[W_{\alpha, \alpha^{\prime}}+\sum_{\beta} \frac{W_{\beta, \alpha^{\prime}} W_{\alpha, \beta}}{E_{0}-E_{\beta}}\right]=0
\end{gathered}
$$

Thus we see that the rôle of 6-body states is just to renormalize the interaction between 2-body and 4-body ones, and for the rest they may be forgotten about. If $E_{0}$ is outside the continuum of excitations, as we shall show below, the corrections are finite, and experience with clusters suggests that they are small. Had we included 8-body excitations, we could have eliminated them by solving the system for their coefficients and substituting, thus reducing to the above problem with further renormalizations. In principle, the method applies to all the higher order interactions, and we can recast our problem as if only 2 and 4-body states existed. Again, the $W_{\alpha^{\prime}, \alpha}$ term can be eliminated by taking linear combinations of the $\alpha$ states: when this is done, we get a self-energy correction to $E_{\alpha}$ and a mixing of the $W_{m, \alpha}$ vertices. The equations become

$$
\begin{gathered}
\left(E_{m}-E_{0}\right) a_{m}+\sum_{m^{\prime}} a_{m^{\prime}} W_{m, m^{\prime}}+\sum_{\alpha} b_{\alpha} W_{m, \alpha}=0 \\
\left(E_{\alpha}-E_{0}\right) b_{\alpha}+\sum_{m^{\prime}} a_{m^{\prime}} W_{\alpha, m^{\prime}}=0
\end{gathered}
$$

Solving for $b_{\alpha}$ and substituting in the first equation we exactly decouple the 4-body states as well. The eigenvalue problem is now

$$
\left(E_{0}-E_{m}\right) a_{m}=\sum_{m^{\prime}} a_{m^{\prime}}\left\{W_{m, m^{\prime}}+\left\langle m\left|S\left[E_{0}\right]\right| m^{\prime}\right\rangle\right\},
$$

where

$$
\left\langle m\left|S\left[E_{0}\right]\right| m^{\prime}\right\rangle=\sum_{\alpha} \frac{<m|W| \alpha><\alpha|W| m^{\prime}>}{E_{0}-E_{\alpha}} .
$$

Equation (72) is of the form of a Schrödinger equation with eigenvalue $E_{0}$ for pairs with an effective interaction $W+S$. Then we interpret $a_{m}$ as the wave function of the dressed pair, which is acted upon by an effective Hamiltonian $\tilde{H}$. The change from the full many-body $\mathrm{H}$ to $\tilde{H}$ is the canonical transformation which generalizes the one of the previous Section to all orders. In the 
new picture, the holes interact through an effective vertex with infinitely many contributions, some of which are shown in Figure 3. The linked contributions represent repeated exchange of electron-hole pairs, and may contain self-energy insertions; all these contributions make up the effective interaction. The unlinked diagrams are pure self-energy. Thus, the scattering operator $S$ is of the form $S=W_{\text {eff }}+F$, where $W_{\text {eff }}$ is the effective interaction between dressed holes, while $F$ is a forward scattering operator, diagonal in the pair indices $m, m^{\prime}$ which accounts for the self-energy corrections of the one-body propagators: it is evident from (72) that it just redefines the dispersion law $E_{m}$, and, essentially, renormalizes the chemical potential. Therefore $F$ must be dropped, as in Cooper theory 22 and in the last Section.

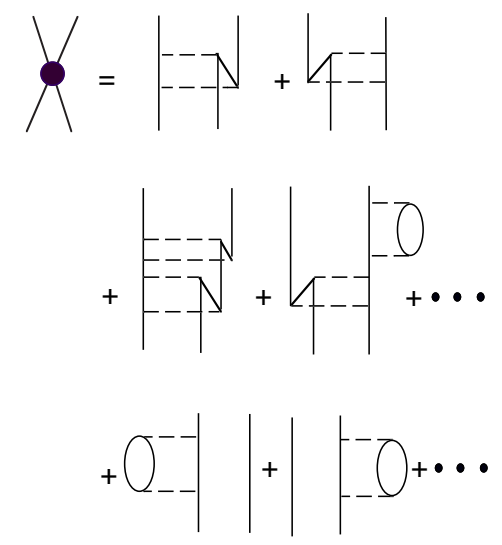

FIG. 3. Some terms of the expansion of the effective hole-hole vertex (blob) defined by $\tilde{H}$. The dashed lines represent $W$ interactions. Linked diagrams belong to $\tilde{W}_{e f f}$, and unlinked ones to $F$.

So, the effective Schrödinger equation for the pair reads

$$
\left(H_{0}+W+W_{e f f}\right)\left|a>=E_{0}\right| a>
$$

and we are interested in the possibility that $E_{0}=2 E_{F}-$ $|\Delta|$, with a positive binding energy $|\Delta|$ of the pair. Any other pairing mechanism not considered here, like off-site interactions, inter-planar coupling and phonons, can be included as an extra contribution to $W_{m^{\prime}, m}$ which just adds to $W_{\text {eff }}$.

We emphasized the fact that the canonical transformation is exact because in this way our argument does not require $\mathrm{U} / \mathrm{t}$ to be small. In the numerical calculations below, we shall drop $W$ and approximate $W_{\text {eff }}$ neglecting the 6-body and higher excitations; in other terms, we shall compute the bare quantities. At least the structure of the solution is exact when expressed in terms of renormalized matrix elements, and the process can be systematically improved. The $\alpha$ states are those of Equation (53) and the interaction matrix element is given in Equation (55). Working out (73) we find that the product in the numerator yields 4 terms; two are proportional to $\delta(p-s)$ and belong to $F$, while the cross terms yield identical contributions to $W_{\text {eff }}$. Using (32,33), we obtain the effective interaction between $\mathrm{W}=0$ pairs:

$$
\begin{array}{r}
\left\langle\Phi_{\eta}[p]\left|W_{\text {eff }}\right| \Phi_{\eta}[s]\right\rangle= \\
4 \sum_{R \in C_{4 v}} \chi^{(\eta)}(R) \sum_{k}^{o c c} \theta\left(\varepsilon(R s+p+k)-E_{F}\right) \\
\frac{U(R s+p+k,-p, R s, k) U(p, k, R s+p+k,-R s)}{\varepsilon(R s+p+k)-\epsilon(k)+\epsilon(s)+\epsilon(p)-E_{0}}
\end{array}
$$

The sum is over occupied $k$ with empty $R s+p+k$. Note that $W_{\text {eff }}$ does not depend on the sign of $U$. We may see that the perturbation theory of Section $\mathrm{V}$ yields the arithmetical mean of the two unperturbed limits $E_{0} \rightarrow 2 \varepsilon(p)$ and $E_{0} \rightarrow 2 \varepsilon(s)$. The diagonal elements of $(75)$ are also clearly related to the $\Delta^{(2)}$ expression derived from perturbation theory for the fully symmetric clusters [15], which are special cases of the present theory. The expressions (5), (58) and (75) are characterized by the symmetry-induced quantum mechanical interference of several terms. The sum can be positive or negative depending on the Irrep. This interference produces a partial cancellation, and the absolute value of the result is typically much smaller than individual contributions. This explains why the interaction is dynamically small for $\mathrm{W}=0$ pairs: they have no direct interaction, and because of the interference the effective interaction is reduced compared to what one could expect by a rough order-of-magnitude estimate. However, the presence of the $\theta$ functions and the anisotropy of the integrands prevent a total cancellation.

In (5), the vanishing of the denominators is prevented by the discrete spectrum of the cluster; however, when applying (58) to the plane the integrand is singular. This complication disappears in the full expression $(75)$ since we are interested in bound pairs; then, $E_{0}$ is below the continuum and the denominators never vanish.

The $s$ and $p$ indices run over $1 / 8$ of the BZ. We denote such a set of empty states $e / 8$, and cast the result in the form of a (Cooper-like) Schrödinger equation

$$
2 \varepsilon(k) a(k)+\sum_{k^{\prime}}^{e / 8} W_{e f f}\left(k, k^{\prime}\right) a\left(k^{\prime}\right)=E_{0} a(k)
$$

for a self-consistent calculation of $E_{0}$ (since $W_{\text {eff }}$ depends on the solution). Let $N_{C}$ be the number of cells in the crystal. The $U$ matrix elements (28,29) scale as $N_{C}^{-1}$ and therefore $W_{\text {eff }}$ scales in the same way. For an infinite system, $N_{C} \rightarrow \infty$, this is a well defined integral equation. The existence of a clear-cut continuum limit means that the problem is well posed, but this equation is very difficult to treat analytically. The interaction matrix is complicated by the Umklapp discontinuities, other 
discontinuities come from the limitations to occupied or empty states, and there are several independent variables since everything is anisotropic. Therefore, we must resort to a numerical treatment. For the sake of simplicity, we shall neglect the minor contributions from the higher bands and consider the dominant intra-band processes, in which empty states belong to the bonding band.

\section{SUPERCELL CALCULATIONS}

Several good supercell calculations devoted to the problem of pairing have been reported to date [23], but no conclusive evidence was reached, because of the difficulty of dealing with size effects. Any brute force approach will miss the result by far, because size effects are quite important, or if you wish, the minimum size useful for simulating the plane is enormous. Now we have the advantage of starting from an analytic result (Equation (75)) which holds for the full plane. However the analytic solution is difficult, so we must discretise the problem. The best way is to work with a supercell of $N_{S C} \times N_{S C}=N_{C}$ cells, with periodic boundary conditions. The advantage remains, however, because Equation (75), however hard, is easy compared to direct diagonalisation. We are able to solve the problem in a virtually exact way for $N_{C}=30$ or 40 , while the calculation becomes somewhat hard with larger supercells.

We loooked for triplet bound states, but we did not find them, because, as in the clusters, $W_{e f f}$ is repulsive for triplets. Therefore the rest of the analysis is concerned with the $\mathrm{W}=0$ singlets, which do show pairing, as we demonstrate below.

The $\Delta<0$ behaviour that we obtain in supercell calculations is in line with our previous findings in small clusters ( 11], 15], 116], 17], 18). The supercells have periodic boundary conditions instead of the open boundary conditions we were using previously and are very much larger systems than those that one can afford by exact diagonalisation. However, we are interested in the asymptotic behavior for $N_{C} \rightarrow \infty$ and $\Delta$ depends on $U^{\prime} s$ and $N_{C}$ in a peculiar way, within the range of attainable supercell sizes. It increases with $N_{C}$ for large $U^{\prime} s$ and drops for small $U^{\prime} s$; we must understand the dependence in order to be able to make reliable extrapolations. Cooper [22] assumed a constant negative interaction $-V_{C}$ within a distance $\omega_{D}$ from $E_{F}$, where $\omega_{D}$ is the Debye frequency. His result is well known:

$$
\left|\Delta_{C}\right|=\frac{2 \omega_{D}}{e^{\frac{1}{V_{C} \rho_{F}}}-1}
$$

where $\rho_{F}$ is the density of states at the Fermi level. The orders of magnitude are $\omega_{D} \approx 25 \mathrm{meV}, \rho_{F} \approx$ $0.3 \mathrm{eV}^{-1} / \mathrm{cell}$ and $V_{C} \approx 1 \mathrm{eV} / \mathrm{cell}$, and $\left|\Delta_{C}\right|$ is in the meV range. Here, no $\omega_{D}$ is involved, and all the empty states contribute; however, we recall that all the distinct $W=0$ pairs may be labeled from the $k$ points in the $e / 8$ set. We define the Uniform Interaction Model (UIM) in which a constant negative interaction $-V$ prevails for $k$ and $k^{\prime}$ in $e / 8$. Both the Cooper and the UIM models can be solved in supercell calculations, and show the same qualitative behavior with increasing $N_{C}$. For small supercells, the binding energy is of the same order as $\mathrm{V}$, while for large cells it tends to the asymptotic value $-\Delta_{\text {asympt }}$, which may be very different. This is similar to the behavior we observe in the Hubbard model. Moreover, we can compute $\Delta$ for a given filling and $N_{S C}$ according to $(76)$, and determine the value of $V$ which gives the same $\Delta$ in the UIM. This will be $V_{\text {eff }}$, that is, the effective $V$ of our theory, characterizing the strength of the attraction by a single quantity. Fortunately, $V_{\text {eff }}$ does not depend too much on the supercell size,and is seen to converge with increasing $N_{S C}$.

We use as input data the current estimates (in eV) $\mathrm{t}=1.3, \varepsilon_{p}=3.5, \varepsilon_{d}=0, U_{p}=6 s, U_{d}=5.3 s$, where $\mathrm{s}$ is a scale factor induced by renormalization. At $\mathrm{s}=2.121$, with $E_{F}=-1.3 \mathrm{eV}$, we get for ${ }^{1} B_{2}$ pairs the results shown in Table II. Here, $n_{\text {tot }}$ is the filling and $V_{\text {ef } f}$ is derived by comparison with UIM calculations. We see that although $\Delta \mid$ decreases monotonically with increasing supercell size up to $N_{S C}=40, V_{\text {eff }}$ is fairly stable and is clearly not dropping to zero; it corresponds to $\left|\Delta_{\text {asympt }}\right|$ values of about $20 \mathrm{meV}$.

In addition, the UIM can be solved in the thermodynamic limit; writing $E_{0}=2 E_{F}-|\Delta|$ we can estimate $\Delta_{\text {asympt }}$ for $N_{C} \rightarrow \infty$. Indeed, the Cooper-like Schrödinger equation (76) with $W_{\text {eff }}=-\frac{V}{N_{C}}, V>0$, leads to

$$
\frac{8}{V}=\int_{E_{F}}^{0} \frac{d \epsilon \rho(\varepsilon)}{2\left(\varepsilon-E_{F}\right)+\left|\Delta_{\text {asympt }}\right|}
$$

where $\rho$ is the density of states. This is readily solved numerically. The dependence of $\Delta$ on $\mathrm{V}$ is qualitatively similar to the dependence of $\Delta_{C}$ on $V_{C}$, and good fits can be obtained using the Cooper formula with effective $\omega_{D}$ and $\rho_{F}$ parameters, like those shown in Table III.

\section{DOPING DEPENDENCE}

In Table IV, we consider ${ }^{1} B_{2}$ pairs at $E_{F}=-1.2 \mathrm{eV}$ (with the current parameters, half filling corresponds to $E_{F}=-1.384 \mathrm{eV}$, so the filling we are considering is $n_{\text {tot }} \approx 1.3$ at $N_{S C}=30$ ). We report values of $\Delta$ and $V_{e f f}$ as obtained from supercell calculations at $N_{S C}=12,20$ and 30 for 3 values of the scale factor s. The extrapolation to the infinite supercell is obtained from the UIM with the $V_{\text {eff }}$ value obtained at $N_{S C}=30$. We see that the relatively mild $N_{S C}$ dependence of $V_{\text {eff }}$ supports the use of the UIM to extrapolate the results to the thermodynamic limit, and there is a clear indication of pairing with sizable binding energies. The $s$ dependence of $V_{e f f}$ is roughly linear, while $\Delta$ depends exponentially on $s$. Table $\mathrm{V}$ presents the results for ${ }^{1} B_{2}$ pairs at $E_{F}=-1.1$ 
$\mathrm{eV}\left(n_{t o t} \approx 1.4\right.$ at $\left.N_{S C}=30\right)$. The trend is similar, but $V_{\text {eff }}$ is seen to increase with doping. Tables VI and VII show the results for the ${ }^{1} A_{2}$ pairs. These are seen to lead to bound states as well, with comparable $\Delta$ values; the trend with doping is opposite, however, and the gap is nearly closing at $E_{F}=-1.1 \mathrm{eV}$. A necessary condition for superconducting flux quantization is that two kinds of pairs of similar binding energy and different symmetries exist 19]. A similar conclusion was reached independently by other Authors [14]. Moreover, evidence of mixed $(s+i d)$ symmetry for the pairing state has been amply reported in angle-resolved photoemission studies [24]. This remark leads to the prediction that in this model superconductive pairing disappears with increasing $n_{t o t}$, while a different sort of pairing prevails; in reality the $\mathrm{Cu}-\mathrm{O}$ plane prefers to distort at excessive doping, and in a distorted plane the present mechanism, based on symmetry, could be destroyed.

\section{CONCLUSIONS}

We propose a general framework for the effective interaction between two holes, based on the three-band Hubbard model but ready to include extra interactions as well. An effective Hamiltonian can in principle be obtained by a systematic canonical transformation including any kind of virtual intermediate states. We obtained the closed-form analytic expression of the effective interaction including 4-body virtual states. This describes repeated exchange of an electron-hole pair. The argument does not depend on perturbation theory, and the equations retain their form, with renormalized parameters, at all orders. The previous exact-diagonalisation results of cluster calculations are special cases. The resulting integral Equation (76), with the effective interaction(75), is valid for the full plane. Since an analytic treatment is prohibitive, we resort to a numerical treatment by supercell calculations. Even so, the solution is hard. Depending on the parameters, extremely large supercells may be needed to obtain the convergence of the pair energy $\Delta$ to the bulk limit; however, we find that precisely the same effect occurs in the Uniform Interaction Model where a constant effective interaction $V$ is assumed. We define $V_{\text {eff }}$ as the value of $V$ that inserted in the UIM yields the same $\Delta$ in supercell calculations as our integral equation. Since $V_{\text {eff }}$ converges to the bulk value much more readily, we are able to go to the asymptotic limit, and to show the instability of the Fermi liquid in the model at hand. $\Delta_{\text {asympt }}$ values in the range of several tens to a few hundreds of meV are obtained if we multiply the $U$ parameters by a scale factor $\mathrm{s}$ which is somewhat larger than 1 . The values $U_{d}=5.3 \mathrm{eV}, U_{p}=6$. eV differ appreciably from other literature estimates [25], and must depend on the compound and doping. For example, in $\mathrm{La}_{2} \mathrm{CuO}_{4}, U_{p}=4 \mathrm{eV}$ and $U_{d}=10.5 \mathrm{eV}$ have been recommended [26]. However, since the screening excitations are explicitly accounted for in the Hamiltonian, it is reasonable that the input $U^{\prime}$ s must be somewhat larger than the fully screened interaction. Moreover, contributions from phonons and other mechanisms can be included as additive terms of $W$, and must be relevant for a comparison with experiment. We find that ${ }^{1} A_{2}$ pairs are more tightly bound close to half filling, but ${ }^{1} B_{2}$ pairs are favored when the filling increases. We remind the reader here that these symmetry labels are not absolute, but depend on the choice of a gauge convention [27]. We get attraction and pairing at all fillings, but the binding energy of the ${ }^{1} A_{2}$ pairs drops by orders of magnitude as the filling increases; thus, there is no chance of superconducting flux quantization too far from half filling. So, we do not predict that superconductivity occurs outside some range of hole concentration. However, pairing is still there, even for large doping: since the present mechanism is driven by symmetry it works unless the system distorts. In fact, at excessive dopings the real superconductors develop stripes, and become normal metals. The three-band Hubbard model might be too idealized to allow a detailed comparison with experiments; however we stress that the approach presented is far more general than the model we are using, and can be applied to more realistic Hamiltonians. This is the main result of the present paper.

This work has been supported by the Istituto Nazionale di Fisica della Materia. We gratefully acknowledge A. Sagnotti, Università di Roma Tor Vergata, for useful and stimulating discussions.

[1] J.G. Bednorz and K.A. Muller, Z. Phys. B64 (1986) 189.

[2] L. Pietronero and S. Strässer, Europhys. Lett18, 627 (1992).

[3] G. Iadonisi,M. Chiofalo, V. Cataudella and D. Ninno, Phys. Rev. B48, (1993) 12966 and references therein.

[4] J.R.Schrieffer, X.C.Wen and S.C.Zhang, Phys. Rev. Letters, 60, 944 (1988).

[5] P.W. Anderson, Science,235,(1987),1196; see also The Hubbard Model, Ed. D. Baeriswyl et al., Plenum Press, New York, 1995, page 217.

[6] O.P. Sushkov, Phys. Rev. B54, 9988 (1996) and reference therein; V.V. Flambaum, M.Yu. Kuchiev and O.P. Sushkov, Physica C 235-240, 2218 (1994).

[7] J.E.Hirsch,S.Tang, E.Loh Jr, and D.J.Scalapino, Phys. Rev.Letters 60, 1668 (1988); Phys. Rev. B39, 243 (1989).

[8] C.A.Balseiro, A.G.Rojo, E.R.Gagliano, and B.Alascio, Phys.Rev.B 38, 9315 (1988).

[9] D.H. Jones and S.J.Monaghan, J. Phys. Condens. Matter, 1 (1989) 1843.

[10] S. Mazumdar, F. Guo, D. Guo, K.C. Ung and J. Tinka Gammel, Proc. of the Discussion Meeting on Strongly Correlated Electron Systems in Chemistry, Bangalore, India (Springer Verlag, Berlin 1996). 
[11] M. Cini and A. Balzarotti, Il Nuovo Cimento D 18, 89 (1996).

[12] M.A. Van Veenendal and G.A. Sawatzky, Phys. Rev. Lett. 70, 2459 (1993).

[13] M. Schluter, in Superconductivity and Applications, edited by H.S. Kwok et al. (Plenum, New York, 1990),p.1.

[14] H.-B. Schüttler, C. Gröber,H.G. Evertz and W. Hanke, cond-mat/9805133

[15] M. Cini and A. Balzarotti, Phys. Rev. B56, 1, 14711 (1997).

[16] M. Cini and A. Balzarotti, J. Phys. Condens. Matter8 , L265 (1996).

[17] M. Cini and A. Balzarotti, Solid State Commun.101, 671 (1997).

[18] M. Cini, A. Balzarotti, J. Tinka Gammel and A. R. Bishop, Nuovo Cimento 19 D, (1997) 1329.

[19] M. Cini, A. Balzarotti and G. Stefanucci, submitted for publication.

[20] M. Cini, A. Balzarotti and G. Stefanucci, condmat/9808209 and submitted for publication.

[21] Raimundo R. dos Santos, cond-mat/9502043.

[22] L.Cooper, Phys. Rev. B104, 1189 (1956); C. Kittel, Quantum Theory of Solids, John Wiley and Sons, New York (1963) Chapter 8.

[23] E. Dagotto, Rev. Mod. Phys. 66 1324, (1994) 763.

[24] Z.X. Shen, W.E. Spicer, D.M. King, D.S. Dessau and B.O. Wells, Science 267, 343 (1995) and References therein.

[25] A.K. McMahan, R.M. Martin and S. Satpathy, Phys. Rev. B 38, 6650 (1988).

[26] M. S. Hybertsen, E.B. Stechel, M. Schluter and D. R.Jennison, Phys. Rev. B41, 11068 (1990).

[27] In comparing these results with those of various calculations from other Authors, one should bear in mind that in the three-band Hubbard model for the $\mathrm{Cu}-\mathrm{O}$ plane different conventions have been widely used in the literature for the phase of orbitals, which are equally possible because of the gauge invariance of the theory. We are using a model Hamiltonian (2), which is simplest because a $\mathrm{Cu}$ ion is bonded to the nearest neighbor $\mathrm{O}$ by identical hopping integrals $t$; however, various Authors prefer an alternating bond convention, with hopping integrals changing sign for a $90^{\circ}$ rotation. With to the choice of the gauge, the symmetry labels vary, according to the multiplication table of Irreps of the $C_{4 v}$ Group.

\begin{tabular}{lllclcl}
\hline \hline $\mathrm{C}_{4 v}$ & $\mathrm{E}$ & $\mathrm{C}_{2}$ & $2 \mathrm{C}_{4}$ & $2 \sigma$ & $2 \sigma^{\prime}$ & \\
$\mathrm{A}_{1}$ & 1 & 1 & 1 & 1 & 1 & \\
$\mathrm{~A}_{2}$ & 1 & 1 & 1 & -1 & -1 & $R_{z}$ \\
$\mathrm{~B}_{1}$ & 1 & 1 & -1 & 1 & -1 & $x^{2}-y^{2}$ \\
$\mathrm{~B}_{2}$ & 1 & 1 & -1 & -1 & 1 & $x y$ \\
$\mathrm{E}$ & 2 & -2 & 0 & 0 & 0 & $(x, y)$ \\
\hline \hline
\end{tabular}

TABLE I. The Character Table of the $C_{4 v}$ Group

\begin{tabular}{lclcl}
\hline \hline$N_{S C}$ & $n_{\text {tot }}$ & $-\Delta(\mathrm{meV})$ & $V_{\text {eff }}(\mathrm{eV})$ & $\Delta_{\text {asympt }}(\mathrm{meV})$ \\
18 & 1.13 & 121.9 & 7.8 & 41.6 \\
20 & 1.16 & 42.2 & 5. & 9.0 \\
24 & 1.14 & 59.7 & 7. & 28.9 \\
30 & 1.14 & 56. & 5.7 & 13.2 \\
40 & 1.16 & 30.5 & 6.6 & 23.4 \\
\hline \hline
\end{tabular}

TABLE II. Binding Energy of ${ }^{1} B_{2}$ Pairs in supercells.

\begin{tabular}{lcl}
\hline \hline$E_{F}(\mathrm{eV})$ & $\omega_{D}(\mathrm{eV})$ & $\rho_{F}\left(\mathrm{eV}^{-1} / \mathrm{Cell}\right)$ \\
-1.35 & 0.4545 & 0.0486 \\
-1.3 & 0.492 & 0.0415 \\
-1.2 & 0.520 & 0.0338 \\
-1.1 & 0.5237 & 0.02905 \\
& &
\end{tabular}

TABLE III. Effective Cooper parameters for fitting the $\mathrm{V}$ dependence of $\Delta$ at several $E_{F}$ values.

\begin{tabular}{lcccccl}
\hline \hline & $s=$ & $\sqrt{2}$ & $s=$ & $\frac{3}{\sqrt{2}}$ & $s=$ & $2 \sqrt{2}$ \\
$N_{S C}$ & $-\Delta(\mathrm{meV})$ & $V_{\text {eff }}$ & $-\Delta(\mathrm{meV})$ & $V_{\text {eff }}$ & $-\Delta(\mathrm{meV})$ & $V_{\text {eff }}$ \\
12 & 126 & 6.75 & 251. & 10. & 469. & 14.23 \\
20 & 63. & 6.9 & 135. & 10.6 & 320.5 & 15.9 \\
30 & 27. & 6.3 & 198. & 13.8 & 460. & 20.8 \\
$\infty$ & 8.8 & 6.3 & 131. & 13.8 & 333. & 20.8 \\
\hline \hline
\end{tabular}

TABLE IV. Data for ${ }^{1} B_{2}$ pairs at $E_{F}=-1.2 \mathrm{eV} ; V_{\text {eff }}$ is in $\mathrm{eV}$.

\begin{tabular}{lclcccc}
\hline \hline & $s=$ & $\sqrt{2}$ & $s=$ & $\frac{3}{\sqrt{2}}$ & $s=$ & $2 \sqrt{2}$ \\
$N_{S C}$ & $-\Delta(m e V)$ & $V_{\text {eff }}$ & $-\Delta(\mathrm{meV})$ & $V_{\text {eff }}$ & $-\Delta(\mathrm{meV})$ & $V_{\text {eff }}$ \\
12 & 163. & 9. & 409. & 15.7 & 688. & 22.1 \\
20 & 151. & 10.8 & 371. & 17.6 & 635.5 & 24.3 \\
30 & 80. & 10.3 & 270. & 17.66 & 520. & 24.9 \\
$\infty$ & 35.6 & 10.3 & 170.8 & 17.66 & 362. & 24.9 \\
\hline \hline
\end{tabular}

TABLE V. Data for ${ }^{1} B_{2}$ pairs at $E_{F}=-1.1 e V ; V_{e f f}$ is in $\mathrm{eV}$. 


\begin{tabular}{lcccccc}
\hline \hline & $s=$ & $\sqrt{2}$ & $s=$ & $\frac{3}{\sqrt{2}}$ & $s=$ & $2 \sqrt{2}$ \\
$N_{S C}$ & $\Delta(m e V)$ & $V_{\text {eff }}$ & $\Delta(m e V)$ & $V_{\text {eff }}$ & $\Delta(m e V)$ & $V_{\text {eff }}$ \\
12 & 141. & 7.2 & 274. & 10.43 & 423. & 13.4 \\
20 & 46.2 & 6.5 & 96. & 9.1 & 158.2 & 11.4 \\
30 & 25.9 & 6.23 & 64.8 & 8.8 & 131. & 11.6 \\
$\infty$ & 8.35 & 6.23 & 34. & 8.8 & 81.7 & 11.6 \\
\hline \hline
\end{tabular}

TABLE VI. Data for ${ }^{1} A_{2}$ pairs at $E_{F}=-1.2 \mathrm{eV} ; V_{e f f}$ is in $\mathrm{eV}$.

\begin{tabular}{lclclcl}
\hline \hline & $s=$ & $\sqrt{2}$ & $s=$ & $\frac{3}{\sqrt{2}}$ & $s=$ & $2 \sqrt{2}$ \\
$N_{S C}$ & $\Delta(m e V)$ & $V_{\text {eff }}$ & $\Delta(\mathrm{meV})$ & $V_{\text {eff }}$ & $\Delta(\mathrm{meV})$ & $V_{\text {eff }}$ \\
12 & 72. & 5.5 & 144. & 8.4 & 228. & 11. \\
20 & 10. & 2.4 & 26.5 & 4.38 & 61. & 6.78 \\
30 & 5.48 & 2.5 & 19. & 5.8 & 45. & 8.16 \\
$\infty$ & 1.5 & 2.5 & 2.6 & 5.8 & 14.4 & 8.16 \\
\hline \hline
\end{tabular}

TABLE VII. Data for ${ }^{1} A_{2}$ pairs at $E_{F}=-1.1 \mathrm{eV} ; V_{\text {eff }}$ is in $\mathrm{eV}$. 